\title{
An Improved Normalized Model of Electrical Capacitance Tomography
}

\author{
Yan Jiangbao ${ }^{1,2, *}$, Zhou Lei $^{2,3}$, Zhu Feng ${ }^{3}$, Tan Xiangyu ${ }^{2}$,and Zhang Changsheng ${ }^{1}$ \\ ${ }^{1}$ College of Information Engineering and Automation, Kunming University of Science and Technology, Kunming, 650500, China; \\ ${ }^{2}$ Yunnan Electric Power Research Institute, Kunming, 650217, China \\ ${ }^{3}$ Department of Automation, North China Electric Power University, 071003, China
}

\begin{abstract}
Electrical capacitance tomography (ECT) technology is a process tomography, which is based on medical CT technology and develops together with computer and sensor technology. The measured capacitances by ECT system are usually normalized at the high and low permittivity for image. Parallel normalization model is commonly used during normalization process, which assumes the distribution of materials in parallel. And thus, the normalized capacitance is a linear function of measured capacitance. The usage of series normalization model results in the normalized capacitance as a nonlinear function of measured capacitance. The research on parallel, series normalization model and hybrid normalization model. In this paper, we propose a method based on the center electric field line to determine the model weighting factor for the hybrid normalization model. The simulation and static test results show that the reconstructed image quality is improved obviously by the hybrid normalization model based on the center electric field line.
\end{abstract}

\section{Introduction}

Electrical capacitance tomography (ECT) is a nonintrusive and non-destructive image reconstruction technique in industrial processes for obtaining information about the contents of closed pipes or vessels by measuring variations in the dielectric property of material inside the vessel. This technique determines the permittivity distribution using capacitance measurements obtained by applied voltages through the electrodes mounted on the periphery of the domain of interest. One can also obtain information about volume fraction and velocities of the contents of pipes for a two-phase system. With the aid of ECT, it is possible to image materials such as oils, plastics, image flames and combustion .The ECT system can measure the characteristic parameters of a two-phase fluid consisting of a medium with different dielectric properties. Using the array capacitive sensor, the combination of the various measurements between the electrodes can reflect the multi-phase fluid concentration and the distribution of the tube in the cross-section, the capacitance measurement as the projection data, through a certain image reconstruction algorithm, It is possible to reconstruct the phase distribution of the fluid flowing through the pipe at a certain moment.

The system of ECT consists of three parts: a sensor, a data acquisition and signal processing unit, and an image reconstruction computer, as shown in Fig1. Electrical tomography is one kind of non-intrusive and visible measurement technique. Electrical tomography has wide applications in clinical monitoring and industrial multiphase flow measurement for the advantages of being fast in response, nondestructive and low cost[1].

The system of ECT can measure the characteristic parameters of a two-phase fluid consisting of a medium with different dielectric properties. Using the array sensor, the combination of the various measurements between the electrodes can reflect the multi-phase fluid concentration and the distribution of the tube in the cross-section, the capacitance measurement as the projection data, through a certain image reconstruction algorithm. It is possible to reconstruct the phase distribution of the fluid flowing through the pipe at a certain moment.

Theoretically, the capacitance between the electrodes can be obtained through a Poisson equation, which is given by

$$
\nabla \cdot[\varepsilon(x, y) \nabla \phi(x, y)]=-\rho(x, y)
$$

Where $\varepsilon(x, y)$ is the permittivity distribution in the sensing field, $\phi(x, y)$ is the potential distribution and $\rho(x, y)$ is the charge distribution, which is the source of the electrical field. Since there are no sources, i.e. no charge inside the sensor, (1) can be rewritten as

$$
\nabla^{2} \phi(x, y)+\frac{1}{\varepsilon(x, y)} \nabla \varepsilon(x, y) \nabla \phi(x, y)=0
$$

Where the electric field $E(x, y)=-\nabla \phi(x, y)$.In general, it is impossible to solve (2) for an inhomogeneous permittivity distribution. The 
potential $\phi(x, y)$ can be calculated numerically by applying the finite element method (FEM).

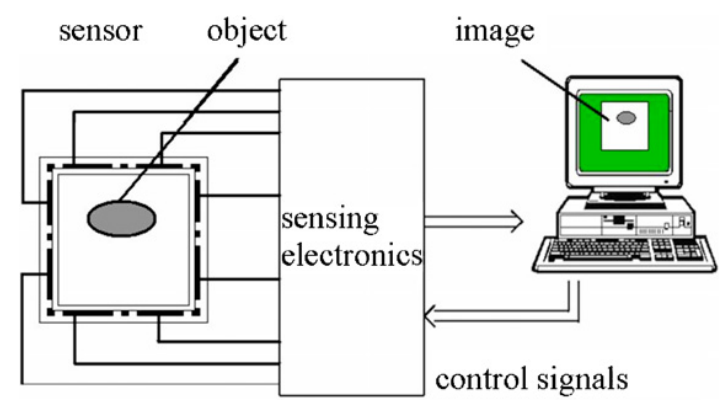

Fig. 1. Layout of the ECT system

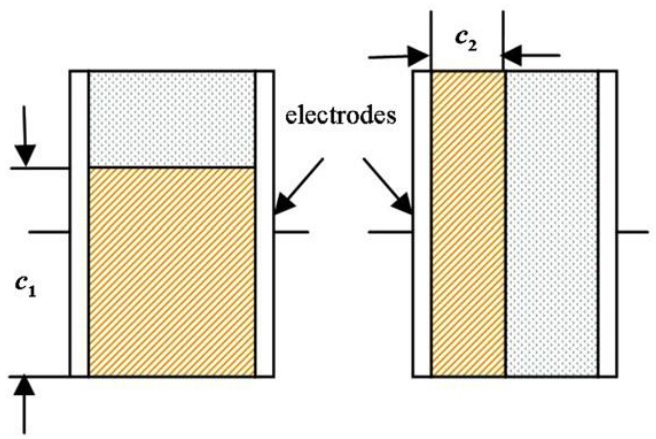

Fig. 2. Normalized model

If the potential $\phi(x, y)$ is known, the charge $Q$ of an electrode can be calculated using Gauss' law[2]:

$$
Q=-\oint_{\Gamma} \varepsilon(x, y) \cdot \nabla \phi(x, y) d \Gamma
$$

Where $\Gamma$ is the electrode surface. Using the charge $Q$ at each detector electrode, the electrical capacitance $C$ can be calculated as

$$
C=\frac{Q}{V}=-\frac{1}{V} \oint_{\Gamma} \varepsilon(x, y) \nabla \phi(x, y) d \Gamma
$$

Where is $V$ the potential difference between the source and detector electrode.

\section{The normalized model}

For the ECT system, the actual measurement is usually carried out empty and full tube calibration, that is, the capacitance value $C^{h}$ and $C^{l}$.For the actual two-phase mixed media, the capacitance measurement data $C^{m}$ should be normalized before calibration using the calibration data. Normalization needs to be met:

$$
\begin{gathered}
\lambda=f\left(C_{m}, C_{h}, C_{l}\right) \\
f\left(C_{m}, C_{h}, C_{l}\right)=0, C_{m}=C_{l} \\
f\left(C_{m}, C_{h}, C_{l}\right)=0, C_{m}=C_{h}
\end{gathered}
$$

The advantage of normalizing the capacitance measurement data is that the measurement data can be dimensionless, facilitated for mathematical processing and associated with digital images and reduced measurement errors. For the same capacitance measurements, different normalized models will affect the quality of the reconstructed image[3].

\subsection{Parallel and Series Normalization Model}

Since the electrode capacitances strongly depend on the sensor electrodes geometric configuration besides permittivity distribution, the capacitance responses between different electrode combinations present a great distinction in magnitude and distribution profile. In order to eliminate the effect and reduce systematic errors in the measurement system, before being applied reconstruction algorithm, the measured capacitances, sensitivity and permittivity in need to be normalized. The parallel and series normalization models are the most widely used in ECT. Both of them treat each pair of measuring electrodes as an ideal parallel plate capacitance sensor, and assume that the distribution of the two types of materials is in parallel or series arrangement. Fig. 1 shows the parallel and series models in (a) and (b) respectively. The oblique portion represents a higher permittivity and the blank portion represents a lower permittivity. $C_{1}$ and $C_{2}$ represent the percentage of thickness for the higher permittivity material.

For the parallel model, the measured capacitance can be expressed as

$$
C^{m}=\left(1-C_{1}\right) C^{l}+C^{h} C_{1}
$$

For the series model,it is given by

$$
1 / C^{m}=1 /\left[\left(1-C_{2}\right) C^{l}\right]+1 /\left(C^{2} C^{h}\right)
$$

Rearranging (8) and (9), $\lambda^{p}$ and $\lambda^{s}$ represent the normalized capacitances in addition to being the volume fraction between the electrode pair $i, j$ corresponding to parallel and series models, and then are given by

$$
\begin{gathered}
C_{1}=\frac{C^{m}-C^{l}}{C^{h}-C^{l}}=\lambda^{p} \\
C_{2}=\left(1 / C^{m}-1 / C^{l}\right)\left(1 / C^{h}-1 / C^{l}\right)=\lambda^{s}
\end{gathered}
$$

Normalizing with $(10)$ is called as conventional normalization model and the normalized capacitance is a linear function of the electrode capacitance, in contrast to the series normalized capacitance being a nonlinear function.

The sensitivity of the electrode pair $i, j$ with respect to the kth element in FEM mesh, an entry of $S$ can be defined as

$$
S_{i, j}(k)=u(k) \cdot \frac{C_{i j}^{m}(k)-C_{i j}^{l}}{C_{i j}^{h}-C_{i j}^{l}} \cdot \frac{1}{\varepsilon_{h}-\varepsilon_{l}}
$$

Where $C_{i j}^{m}(k)$ is the capacitance value between electrode pair $i, j$ when the $k t h$ element is filled with higher permittivity material in the lower permittivity background and $u(k)$ is an area correction factor, which is assigned to the ratio of the area of the largest element to the area of the kth element. 


\subsection{Hybrid normalized model}

Multiphase flow patterns are complex and variable, and for ECT array electrodes, the distribution of capacitance and dielectric between any pair of pairs cannot be simply expressed in parallel or in series model. For the hybrid model, it is given by

$$
\lambda^{c}=(1-\beta) \lambda^{p}+\beta \lambda^{s}=(1-\beta) \frac{C^{m}-C^{l}}{C^{h}-C^{l}}+\beta \frac{1 / C^{m}-1 / C^{l}}{1 / C^{h}-1 / C^{l}}
$$

Where $\lambda^{c}$ the normalized capacitances of hybrid model. $\beta$ is the model weighting factor.

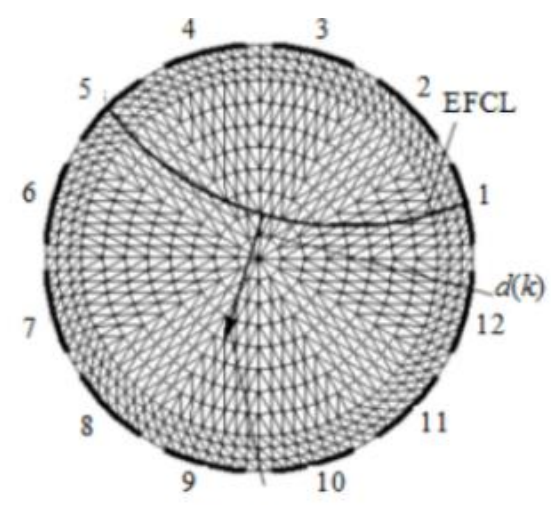

The kthunit

Fig. 3. Electrical field center line between electrode 1 and 5

When $\beta=1$, that is, the series normalization model.

$$
\lambda^{c}=\lambda^{s}=\frac{1 / C^{m}-1 / C^{l}}{1 / C^{h}-1 / C^{l}}
$$

When $\beta=0$, that is, the parallel normalization model.

$$
\lambda^{c}=\lambda^{p}=\frac{C^{m}-C^{l}}{C^{h}-C^{l}}
$$

The hybrid model is the weighted sum of the parallel model and the series model. Sensitivity matrix of hybrid normalized model, it is given by

$$
\begin{array}{r}
S_{i j}(k)=u(k) \cdot\left[(1-\beta(k))\left(\frac{C_{i j}^{m}(k)-C_{i j}^{l}}{C_{i j}^{h}-C_{i j}^{l}}\right)+\right. \\
\left.\beta(k)\left(\frac{1 / C_{i j}^{m}(k)-1 / C_{i j}^{l}}{1 / C_{i j}^{h}-1 / C_{i j}^{l}}\right)\right] \cdot \frac{1}{\varepsilon_{h}-\varepsilon_{l}}
\end{array}
$$

It is necessary to determine the value of $\beta(k)$ for the sensitivity of the split grid of each cell

\section{Model weighting factor calculation}

In this paper, based on electrical field center line method to determine $\beta$,thus determining the hybrid normalized model.The idea of the center electric field line was first proposed by Loser .In this paper, 12 electrode ECT as the object of study, 1-5 electrode center line as shown in Figure 3. Figure 3 shows the distance $d(k)$ between the $k$ unit and the electrical field center line. The model weighting factor is determined using the maximum of the distance between $k$ and each pixel element to the center electric field line. As a result, model weighting factor is formulated as

$$
\beta(x)=\frac{d_{i j}^{k}}{d_{\max }}
$$

Where $d_{i j}^{k}$ is the distance between the $k$ th element and EFCL of the electrode pair $i, j$, and $d_{\max }$ is the maximum distance.

In 1999, Landweber iterative algorithm was first proposed by Yang .Subsequently, Liu et al. Optimized the iterative factors of the algorithm, accelerated the iteration speed, and reconstructed the image[5-6].The parallel normalization model is taken as an example. The algorithm is shown as follows:

$$
\left\{\begin{array}{l}
G_{k+1}=G_{k}+\eta_{k} S^{T}\left(\lambda^{P}-S G_{k}\right) \\
G_{0}=S^{T} \lambda^{P} \\
\eta_{k}=\left\|S^{T} e_{k}\right\|^{2} /\left\|S S^{T} e_{k}\right\|^{2}
\end{array}\right.
$$

Where $e_{k}=\lambda^{p}-S G_{k} . \eta_{k}$ is the $k$ step of the iteration factor. $G_{k}$ is the image gray scale matrix of step $k$.

For the hybrid normalization model, since the normalized capacitance value is related to the model weighting factor, the optimal model weighting factor needs to be determined, and the optimal iteration factor is obtained. At this time, the Landweber iterative algorithm is as follows:

$$
\begin{aligned}
& G_{k+1}=G_{k}+\eta_{k} S^{T}\left(\lambda^{c}-S G_{k}\right) \\
e_{k+1}= & \lambda^{c}-S G_{k+1} \\
= & \lambda^{c}-S\left(G_{k}+\eta_{k} S^{T} e_{k}\right) \\
= & e_{k}-\eta_{k} S S^{T} e_{k} \\
= & \left(I_{M}-\eta_{k} S S^{T}\right)\left[\left(1-\alpha_{k}\right) \lambda^{p}+\alpha_{k} \lambda^{S}-S G_{k}\right]
\end{aligned}
$$

The square of the norm of $e_{k+1}$ is

$$
\begin{aligned}
J(\eta, \alpha) & =\left\|e_{k+1}\right\|^{2} \\
& =\left\|\left(I_{M}-\eta_{k} S S^{T}\right)\left[\lambda^{p}+\alpha_{k}\left(\lambda^{s}-\lambda^{p}\right)-S G_{k}\right]\right\|
\end{aligned}
$$

$M$ is the number of independent measurements

$$
\begin{aligned}
& \text { Let } \frac{\partial J(\eta, \alpha)}{\partial \eta}=0 \text { and } \frac{\partial J(\eta, \alpha)}{\partial \alpha}=0 \text {, there is } \\
& \eta_{k}=\frac{\left\|S^{T}\left[\lambda^{p}+\alpha_{k}\left(\lambda^{s}-\lambda^{p}\right)-S G_{k}\right]\right\|^{2}}{\left\|S S^{T}\left[\lambda^{p}+\alpha_{k}\left(\lambda^{s}-\lambda^{p}\right)-S G_{k}\right]\right\|^{2}} \\
& \alpha_{k}=\left[A^{T} B^{T} B S G_{k}+\left(A^{T} B^{T} B S G_{k}\right)^{T}-\right. \\
& \left.A^{T} B^{T} B \lambda^{P}-\left(A^{T} B^{T} B \lambda^{P}\right)^{T}\right] /\left(2 A^{T} B^{T} B A\right) \\
& \text { Where } A=\lambda^{s}-\lambda^{p}, B=I_{M}-\eta_{k} S S^{T} \text {. }
\end{aligned}
$$




\section{Experimental results and analysis}

In this paper, we use Core i5 Windows $104 \mathrm{G}$ running memory notebook. In Matlab, we write the software package to realize the hybrid model, and realize the functions of finite element method and Landweber iterative algorithm to reconstruct the image. The ECT system with 12 electrodes, 66 independent measurements, the diameter of the pipe is $125 \mathrm{~mm}$, and the finite element grid is 768. In this paper, the Landweber iterative method is used, the iterative factor is chosen as 2, and the reconstructed image is iterated 300 times. The four simulation models are selected as one object, two objects, three objects and five objects. The reconstructed image is shown in Figure 4.

It can be seen from Fig. 4 that the image obtained by the parallel normalization model is blurred, 2, 3 and 5 objects, the imaging effect of the center object is poor and the reconstructed image near the tube wall is connected, and the series model is better. The model obtained by the mixed model is closer to the true object distribution. The model reconstructed by the mixed model is better than the conventional model in the vicinity of the wall and the central region.

To evaluate the performance, the relative image error(RE) and the correlation coefficient (CC) between estimated permittivity vector $\hat{g}$ and the original permittivity vector $g$ are used and defined as[7]

$$
\begin{gathered}
R E=\frac{\|\hat{g}-g\|}{\|g\|} \\
C C=\frac{\sum_{i=1}^{N}(\hat{g}-\hat{g}) \cdot\left(g_{i}-\bar{g}\right)}{\sqrt{\sum_{i=1}^{N}(\hat{g}-\hat{g})^{2} \cdot \sum_{i=1}^{N}\left(g_{i}-\bar{g}\right)^{2}}}
\end{gathered}
$$

Where $\bar{g}$ and $\hat{g}$ are the mean values of $g$ and $\hat{g}$, respectively. A smaller $R E$ and $\mathrm{CC}$ closer to 1 are considered to be better performance indices. It can be seen from Table 1 that the relative error of reconstruction images based on mixed normalization model is smaller than that of parallel and series normalization model.

Static test using the latest development of Tianjin University FPGA dual-mode (capacitance / resistance) imaging system. Using the Landweber iterative algorithm, reconstructing the image as shown in Fig5.

As can be seen from Figure 5, the first distribution of an organic glass rod in the center of the pipe, another plexiglass rod close to the wall. The reconstructed image of the parallel model can not distinguish between two rods. The reconstructed image of the series model roughly distinguishes two rods, but the edges of the image are not clear. The reconstructed image of the mixed model is very clear to distinguish between two plexiglass rods. The second distribution, the mixed model of the reconstructed image effect is much better than the parallel, series model. The object is clearly distinguished and the edges of the image are clear.

Original parallel series

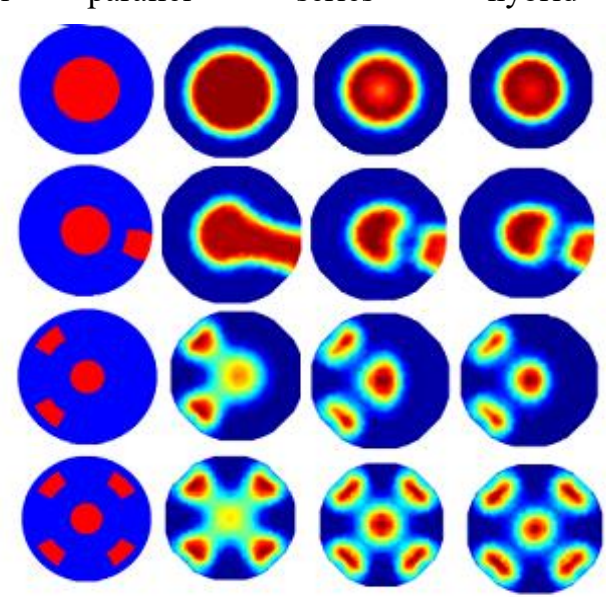

Fig. 4. Reconstructed images

Table 1. Image relative error at 300th iteration

\begin{tabular}{|c|c|c|c|}
\hline Original & Parallel & Series & Hybrid \\
\hline one object & 0.7502 & 0.3134 & 0.2396 \\
\hline two objects & 0.7877 & 0.5368 & 0.4548 \\
\hline three objects & 0.7297 & 0.7104 & 0.6306 \\
\hline five objects & 0.7740 & 0.7707 & 0.6709 \\
\hline
\end{tabular}

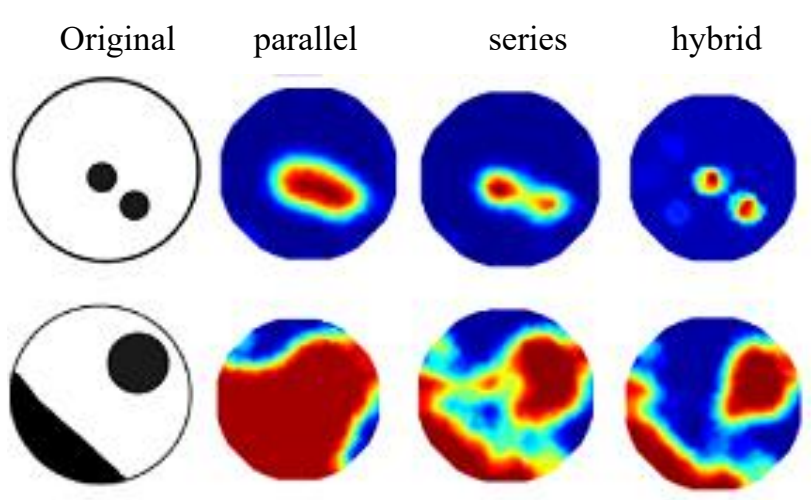

Fig. 5. Static test results

\section{Conclusions}

In this paper, the hybrid normalization model based on the center electric field line is applied to ECt image reconstruction, which improves the quality of image reconstruction. Simulation and results show that the normalized model provides a new method and approach for ECT image reconstruction

In the following research, we propose a method based on the center electric field to determine the model weighting factor of the mixed model. The ECT image reconstruction was performed by using parallel, series and mixed normalization models. Simulation and static tests show that the mixed model can distinguish the 
complex flow pattern of multiple objects, the edge of the image is clear and the relative error is small. It can be seen that the hybrid model is more accurate than the conventional model and can be used as a better equivalent model for the distribution of objects. Simulation results have given superior image quality. It shows that the sensitivity based on the new model is suitable for common iterative algorithms, and achieves a better reconstruction performance.

\section{Acknowledgment}

The author would like to thank Mr. Zhou Lei for his helpful suggestions on the use of ECT DAS during the static experiments.

\section{References}

1. Yong $\mathrm{S} \mathrm{K}$, Seong $\mathrm{H} \mathrm{L}$, Bong $\mathrm{Y} \mathrm{C}$, et al. Sensitivity map generation in electrical capacitance tomography using mixed normalization models[J]. Measurement Science and Technology, 2007, 18 (7):2092-2102.

2. Y.T. Makkawi, P.C. Wright, luidization regimes in a conventional Fluidized bed characterized by means of electrical capacitance tomography[J].Chemical Engineering Science , 2002, 14(4):2411-2437
3. D. L. Donoho. "Compressed sensing," IEEE Transactions on Information Theory, 2006, 52, 1289-1306.

4. Dong X Y and Guo S Q. Image reconstruction method for electrical capacitance tomography based on the combined series and parallel normalization model[J]. Measurement Science and Technology, 2008, 19 (4) : 1032-1039.

5. Yang $\mathrm{W}$ Q, Peng $\mathrm{L} H$. Image reconstruction algorithms for electrical capacitance tomography[J]. Measurement Science \& Technology, 2003, 14(1):R1-R13.

6. Zhang L F, Wang H X. Identification of oil-gas two-phase flow pattern based on SVM and electrical capacitance tomography technique[J]. Flow Measurement \& Instrumentation, 2010, 21(1) : 20-24.

7. Yang WQ, Nguyen T, Betting M, Chondronasios A, Okimoto S, Okimoto FT,McCann H. Imaging wet gas separation process by capacitance tomography.IS\&T/SPIE 14 Symp. Electron Imag 2002. p. 34-58.

8. M. A. T Figueiredo, R. D Nowak, S. J. Wright. "Gradient Projection for Sparse Reconstruction: Application to Compressed Sensing and Other Inverse Problem," IEEE Journal of Selected Topics in Signal Processing, 2007, 1(4): 586-597. 